\title{
A FRAMEWORK FOR LEARNING BIPED LOCOMOTION WITH DYNAMICAL MOVEMENT PRIMITIVES
}

\author{
JUN NAKANISHI ${ }^{1,2}$, JUN MORIMOTO ${ }^{1,2}$, GEN ENDO $^{2,3}$, GORDON CHENG $^{1,2}$, \\ STEFAN SCHAAL ${ }^{2,4}$, and MITSUO KAWATO ${ }^{1,2}$ \\ ${ }^{1}$ Computational Brain Project, ICORP, JST \\ 2-2 Hikaridai, Seika-cho, Soraku-gun, Kyoto, 619-0288, Japan \\ ${ }^{2}$ ATR Computational Neuroscience Laboratories \\ 2-2 Hikaridai, Seika-cho, Soraku-gun, Kyoto, 619-0288, Japan \\ ${ }^{3}$ Sony Intelligence Dynamics Laboratories, Inc. \\ 3-14-13 Higashi-gotanda, Shinagawa-ku, Tokyo, 140-0022, Japan \\ ${ }^{4}$ Dept. of Computer Science and Neuroscience, University of Southern California \\ 3641 Watt way, Los Angeles, CA 90089-2520, USA
}

\begin{abstract}
This article summarizes our framework for learning biped locomotion using dynamical movement primitives based on nonlinear oscillators. Our ultimate goal is to establish a design principle of a controller in order to achieve natural human-like locomotion. We suggest dynamical movement primitives as a central pattern generator (CPG) of a biped robot, an approach we have previously proposed for learning and encoding complex human movements. Demonstrated trajectories are learned through movement primitives by locally weighted regression, and the frequency of the learned trajectories is adjusted automatically by a frequency adaptation algorithm based on phase resetting and entrainment of coupled oscillators. Numerical simulations and experimental implementation on a physical robot demonstrate the effectiveness of the proposed locomotion controller. Furthermore, we demonstrate that phase resetting contributes to robustness against external perturbations and environmental changes by numerical simulations and experiments.
\end{abstract}

Keywords: Biped locomotion, Learning from demonstration, Dynamical movement primitives, Phase resetting, Frequency Adaptation

\section{Introduction}

In this paper, we present an approach to learning biped locomotion from demonstration and its adaptation through coupling between the pattern generator and the mechanical system. Motivated by human's capability of learning and imitating demonstrated movements of a teacher, imitation learning has been explored as an efficient method for motor learning in robots to accomplish desired movements ${ }^{1,2,3}$. In our previous work, we proposed dynamical movement primitives to encode complex discrete and rhythmic multi-joint movements through imitation learning ${ }^{4}$. Dynam- 
ical movement primitives are formulated as a set of autonomous nonlinear differential equations with well-defined attractor dynamics. Demonstrated trajectories are learned using locally weighted regression, and the output of dynamical movement primitives serves as kinematic movement plans, e.g., desired trajectories, for a robot.

This paper presents the idea of using the rhythmic movement primitives based on phase oscillators ${ }^{4}$ as a central pattern generator $(\mathrm{CPG})$ to learn biped locomotion from demonstration. Recently, there has been a growing interest in biologicallyinspired control approaches for rhythmic motion generation using neural oscillators. As a CPG, a neural oscillator model proposed by Matsuoka ${ }^{5}$ is widely used for robotic applications such as biped locomotion ${ }^{6,7,8,9}$, quadruped locomotion ${ }^{10}$, juggling ${ }^{11}$, drumming ${ }^{12}$, and playing with a slinky toy ${ }^{13}$. Neural oscillators have desirable properties such as adaptation to the environment through entrainment. However, it is difficult to design interconnection and feedback pathways of neural oscillators, and much effort is often required to manually tune the parameters of the oscillators in order to achieve the desired behavior.

Compared with neural oscillators, one of the appealing properties of phase oscillators is that the desired phase relationship among oscillators can be specified in a straightforward manner. In ${ }^{14}$, a comprehensive formulation of phase coordination of coupled phase oscillators is proposed. Applications of coupled phase oscillators have been explored in the gait control of multi-legged robots ${ }^{15,16}$ and the control of a biped robot ${ }^{17}$. In addition to using phase oscillators, our movement primitive has various desirable properties which are beneficial for biped locomotion. For example, it can learn a demonstrated trajectory rapidly, and it is easy to re-scale the learned rhythmic movement in terms of amplitude, frequency and offset of the patterns ${ }^{4}$. Furthermore, our movement primitives has the potential capability of improving learned movements through reinforcement learning ${ }^{18}$.

In the application of rhythmic movement primitives to biped locomotion, we introduce coupling terms to the movement primitives to achieve the desired phase relationship among limbs following the formulation proposed in ${ }^{14}$. We also propose an adaptation algorithm for the frequency of walking based on phase resetting ${ }^{19}$ and entrainment between the phase oscillator and mechanical system using feedback from the environment. Phase resetting is introduced to our dynamical movement primitives motivated from a mathematical point of view as well as a biological perspective in order to achieve synchronization of the rhythm of oscillators with the timing of heel strike in biped locomotion. From a mathematical point of view, the phase resetting algorithm can be interpreted as a discretized version of the synchronization mechanism of coupled phase oscillators. From a biological point of view, phenomena of phase resetting or phase shift are observed in many biological oscillators resulting from external perturbations, e.g., circadian pacemakers, biochemical oscillators and human finger tapping neural networks as mentioned in ${ }^{19}$. Phase resetting is related to the stability properties of neural rhythms, which can be analyzed by examining the phase dependent responses against perturbations. A recent 

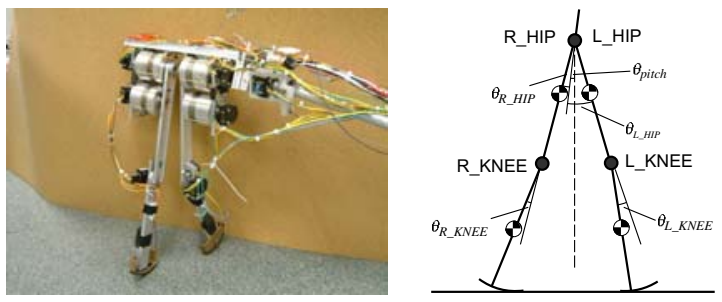

Fig. 1. Left: Physical system. Right: Five-link model of the robot.

work in ${ }^{20}$ studied functional roles of phase resetting for gait stability against external perturbations in biped locomotion. They investigated the behavior of a biped simulation model against external perturbations, where the phase of the prescribed joint trajectories obtained by Fourier fitting of humans' gait data are shifted in response to impulsive forces applied during walking. Their numerical simulations suggest that phase resetting helps maintain stability of periodic movements in biped locomotion in the presence of external perturbations.

In this paper, we demonstrate the effectiveness of the proposed control strategy by numerical simulations and experimental implementation. Furthermore, we evaluate robustness of walking with the proposed algorithm against external perturbations and environmental changes by numerical simulations and experiments.

\section{Experimental Setup}

We use a planar 5-link biped robot developed in ${ }^{21}$ (see Fig. 1). The height of the robot is $0.4 \mathrm{~m}$ and the weight is about $3.4 \mathrm{~kg}$. For numerical simulations, we use the following model: The length of each link of the leg is $0.2 \mathrm{~m}$. The mass of the body is $2.0 \mathrm{~kg}$, the thigh is $0.64 \mathrm{~kg}$ and the shank is $0.05 \mathrm{~kg}$.

The motion of the robot is constrained within the sagittal plane by a tether boom. The hip joints are directly actuated by direct drive motors, and the knee joints are driven by direct drive motors through a wire transmission mechanism with the reduction ratio of 2.0. These transmission mechanisms with low reduction ratio provide high back drivability at the joints. Foot contact with the ground is detected by foot switches. The robot is an underactuated system having rounded soles with no ankles. The robot is controlled with a real-time operating system, RT-Linux. The sampling frequency of the controller is $1 \mathrm{kHz}$.

\section{Biped Locomotion Control with Dynamical Movement Primitives}

In this section, we outline our control framework for biped locomotion using dynamical movement primitives depicted in Fig. 2. 


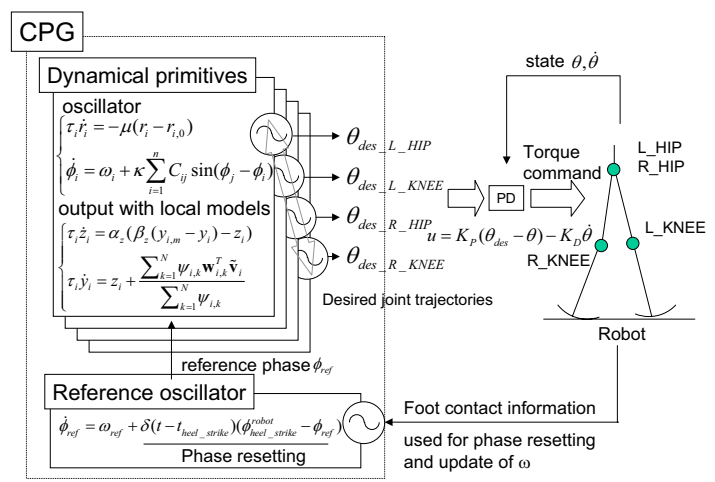

Fig. 2. Proposed control architecture for biped locomotion with dynamical movement primitives.

\subsection{Rhythmic Dynamical Movement Primitives}

Rhythmic dynamical movement primitives encode periodic behavioral patterns as an output of a set of nonlinear dynamical systems composed of a canonical dynamical system with a phase oscillator and a transformation dynamical system with a nonlinear function approximator. We briefly review the formulation of the rhythmic movement primitives we proposed in ${ }^{4}$.

Consider the following limit cycle oscillator characterized in terms of an amplitude $r$ and a phase $\phi$ as a canonical dynamical system which generates basic rhythmic patterns:

$$
\begin{aligned}
\dot{\phi} & =\omega \\
\tau \dot{r} & =-\mu\left(r-r_{0}\right)
\end{aligned}
$$

where $\omega$ is the frequency of the oscillator, $\tau$ is a temporal scaling factor defined by $\tau=1 / \omega, r_{0}$ determines the desired (relative) amplitude, and $\mu$ is a positive constant. When there are multiple oscillators, we will introduce coupling terms among the oscillators (see Section 3.2.1). This rhythmic canonical system is designed to provide an amplitude signal $\tilde{\mathbf{v}}=[r \cos \phi, r \sin \phi]^{T}$ and phase variable $\bmod (\phi, 2 \pi)$ to the following second order transformation dynamical system $(z, y)$, where the output $y$ is used as the desired trajectory for the robot:

$$
\begin{aligned}
\tau \dot{z} & =\alpha_{z}\left(\beta_{z}\left(y_{m}-y\right)-z\right) \\
\tau \dot{y} & =z+f(\tilde{\mathbf{v}}, \phi)
\end{aligned}
$$

where $\alpha$ and $\beta$ are time constants, $y_{m}$ is an offset of the output trajectory. $f$ is a nonlinear function approximator using local linear models ${ }^{22}$ of the form:

$$
f(\tilde{\mathbf{v}}, \phi)=\frac{\sum_{k=1}^{N} \Psi_{k} \mathbf{w}_{k}^{T} \tilde{\mathbf{v}}}{\sum_{k=1}^{N} \Psi_{k}}
$$


where $\mathbf{w}_{k}$ is the parameter vector of the $k$-th local model. Each local model is weighted by a Gaussian kernel function

$$
\Psi_{k}=\exp \left(-h_{k}\left(\bmod (\phi, 2 \pi)-c_{k}\right)^{2}\right)
$$

where $c_{k}$ is the center of the $k$-th linear model, and $h_{k}$ characterizes its width. A final prediction is calculated by the weighted average of the predictions of the individual models. The parameters $\mathbf{w}_{k}$ are determined by locally weighted learning ${ }^{22}$ from a demonstrated trajectory $y_{\text {demo }}$. Given a sampled data point $\left(f_{\text {target }}, \tilde{\mathbf{v}}\right)$ at $t$ where

$$
f_{\text {target }}=\tau \dot{y}_{\text {demo }}-z_{\text {demo }}
$$

and

$$
\tau \dot{z}_{\text {demo }}=\alpha_{z}\left(\beta_{z}\left(y_{m}-y_{\text {demo }}\right)-z_{\text {demo }}\right),
$$

the learning problem is formulated to find the parameters $\mathbf{w}_{k}$ in (5) using incremental locally weighted regression technique ${ }^{22}$ in which $\mathbf{w}_{i}$ is updated by

$$
\mathbf{w}_{k}^{t+1}=\mathbf{w}_{k}^{t}+\Psi_{k} \mathbf{P}_{k}^{t+1} \tilde{\mathbf{v}} e_{k}
$$

where

$$
\begin{gathered}
\mathbf{P}_{k}^{t+1}=\frac{1}{\lambda}\left(\mathbf{P}_{k}^{t}-\frac{\mathbf{P}_{k}^{t} \tilde{\mathbf{v}} \tilde{\mathbf{v}}^{T} \mathbf{P}_{k}^{t}}{\frac{\lambda}{\Psi_{k}}+\tilde{\mathbf{v}}^{T} \mathbf{P}_{k}^{t} \tilde{\mathbf{v}}}\right) \\
e_{k}=f_{\text {target }}-\mathbf{w}_{k}^{T} \tilde{\mathbf{v}}
\end{gathered}
$$

and $\lambda \in[0,1]$ is a forgetting factor. As illustrated in ${ }^{4}$, the amplitude, frequency and offset of the learned rhythmic patterns can be easily modified by scaling the parameters $r_{0}, \omega(=1 / \tau)$ and $y_{m}$ individually.

\subsection{Rhythmic Dynamical Movement Primitives as a CPG}

We use the rhythmic dynamical movement primitives described above as a CPG for biped locomotion. Fig. 2 illustrates the proposed control architecture in this paper. Each joint is equipped with a movement primitive which generates the desired joint trajectory. The output of the movement primitive $y$ is used as the desired trajectory $\theta_{\text {des }}$ for each joint. We define the index and the corresponding name of the joint as Left hip ( $i=1$, L_HIP), and Left knee $(i=2$, L_KNEE), Right hip ( $i=3$, R_HIP), and Right knee $\left(i=4, \mathrm{R} \_\mathrm{KNEE}\right)$. An additional oscillator $\left(\phi_{\text {ref }}\right)$ is allocated to provide a reference phase signal to the limb oscillators, which is adjusted by the ground contact information at the instance of heel strike.

Section 3.2.1 introduces coupling to the oscillators of the movement primitives to achieve the desired phase relationship between the limbs. Section 3.2.2 outlines the phase resetting and frequency adaptation algorithms of the learned periodic movements through the interaction among the coupled oscillators, robot and environment. 


\subsubsection{Inter- and Intra-limb Phase Coordination}

Coupling among the oscillators is introduced to regulate the desired phase relationship between the limbs of the robot. This is motivated from a biological point of view where it has been hypothesized that coupling among neural oscillators plays an important role in coordinating the desired phase relationship of limb movements in locomotion and gait transition ${ }^{23}$.

Consider the following coupling terms for the oscillator $i$ :

$$
\dot{\phi}_{i}=\omega_{i}+\kappa \sum_{i=1}^{N} C_{i j} \sin \left(\phi_{j}-\phi_{i}\right)
$$

where $\kappa$ is a positive constant gain, and $C_{i j}$ is an element of the $n \times n$ matrix $\mathbf{C}$ which characterizes the coupling with other oscillators. This form of coupling appears in various studies of coupled oscillators and their application, e.g., $17,14,15,16,24,25$. Specifically, we employ the formulation in ${ }^{14}$ to coordinate the desired phase relationship. We design the desired phase difference among the canonical oscillators such that the links of each leg move in phase (with zero phase difference), and the left and right legs move out of phase (with $\pi$ phase difference) by defining the phase of the oscillator as $\phi_{i}=0$ at the instance of heel strike of the corresponding leg. More specifically, we require $\phi_{1}-\phi_{2}=0, \phi_{3}-\phi_{4}=0, \phi_{1}-\phi_{3}=\pi$, and $\phi_{2}-\phi_{4}=\pi$. Thus, the connection matrix $\mathbf{C}$ is chosen to be

$$
\mathbf{C}=\left[\begin{array}{cccc}
0 & 1 & -1 & -1 \\
1 & 0 & -1 & -1 \\
-1 & -1 & 0 & 1 \\
-1 & -1 & 1 & 0
\end{array}\right]
$$

\subsubsection{Phase Resetting and Frequency Adaptation of $C P G$}

The phase resetting and frequency adaptation algorithms are motivated by the synchronization mechanism of the coupled oscillators to adjust the frequency of the learned periodic motions by the robot through the interaction among the CPG, robot and environment. In our original formualtion in ${ }^{26}$, phase resetting was directly introduced to all the limb oscillators. Instead, in our current setting, we first introduce phase resetting to the reference oscillator. Then, additional continuous coupling is introduced to the limb oscillators to achieve the desired relative phase to the reference oscillator in order to avoid discontinuity to the desired joint trajectories.

Consider the following phase resetting and frequency update law to the reference oscillator at the instance of heel contact:

$$
\begin{gathered}
\dot{\phi}_{\text {ref }}=\hat{\omega}_{\text {ref }}^{n}+\delta\left(t-t_{\text {heel strike }}\right)\left(\phi_{\text {heel strike }}^{\text {robot }}-\phi_{\text {ref }}\right) \\
\hat{\omega}_{\text {ref }}^{n+1}=\hat{\omega}_{\text {ref }}^{n}+K\left(\omega_{\text {measured }}^{n}-\hat{\omega}_{\text {ref }}^{n}\right)
\end{gathered}
$$


where $\delta$ is the Dirac's delta function, $n$ is the number of steps, and $\phi_{\text {heel }}^{\text {robot }}$ strike is the phase of the mechanical oscillator (robot) at heel strike defined as $\phi_{\text {heel }}^{\text {robtrike }}=0$ at the heel strike of the left leg, and $\phi_{\text {heel }}^{\text {robot }}$ strike $=\pi$ at the heel strike of the right leg. $\omega_{\text {measured }}^{n}$ is the measured frequency of locomotion defined by

$$
\omega_{\text {measured }}^{n}=\frac{\pi}{T_{\text {measured }}^{n}}
$$

where $T_{\text {measured }}^{n}$ is the stepping period of locomotion (half period with respect to the oscillator). At the same time, natural frequencies of all the limb oscillators $\omega_{i}$ are updated at the instance of heel contact such that $\omega_{i}=\hat{\omega}_{r e f}^{n+1}$, and additional coupling is introduced to the limb oscillators with $\phi_{\text {ref }}$ to achieve the desired relative phase $\phi_{1}=\phi_{2}=\phi_{\text {ref }}$ and $\phi_{3}=\phi_{4}=\phi_{\text {ref }}+\pi$.

\section{Numerical Simulations}

\subsection{Learning from Demonstrated Trajectory}

As a demonstrated trajectory, we use the motion capture data of human walking in ${ }^{27}$ (29-year-old male, $173 \mathrm{~cm}, 83.5 \mathrm{~kg}$, right hip and knee). We identified the period and frequency of this pattern by the power spectrum estimation with FFT and autocorrelation as $T=1.17 \mathrm{sec}$ and $f=1 / T=0.855 \mathrm{~Hz}$ respectively. The dynamics of the robot are derived using SD/FAST ${ }^{\mathrm{a}}$ and integrated using the Runge-Kutta algorithm at $1 \mathrm{~ms}$ step size. The ground contact force is calculated using a linear spring-damper model. A low-gain PD controller is used at each joint to track the desired trajectory which is the output of the movement primitive.

A walking pattern from the demonstrated trajectory is learned with the dynamical primitives. We manually designed the desired trajectory for the initial step of locomotion from a standing position at rest, and the proposed CPG controller is activated at heel contact of the first step. The amplitude parameter of the dynamical primitives is set to $r_{0}=0.7$, and the offset $y_{m}=0.375$ is introduced to the knee joints. For the scaling of the natural frequency of the oscillator, the adaptation law described in Section 3.2.2 is used with the initial frequency of $\omega=4.83 \mathrm{rad} / \mathrm{s}$ (period of oscillation is $1.3 \mathrm{sec}$ ). These parameters are determined empirically from trial and error.

Figure 3 illustrates the desired and actual joint trajectories for the left leg, and the timing of heel strike after a stable pattern was learned by the phase resetting algorithm. Figure 4 shows the torque command for the left leg, which indicates that the knee joint swings passively since it requires almost no torque (see $t=15.1 \sim 15.3$ sec). Figure 5 depicts one step of walking. Figure 6 (left) shows the adaptation of the period of locomotion and Fig. 6 (right) shows the learning curve of the frequency of the CPG with different coupling constants $K=0.2,0.5$ and 0.8 in (12). The stepping period approached $0.387 \mathrm{sec}$, and the resultant $\mathrm{CPG}$ frequency was $\omega=8.12 \mathrm{rad} / \mathrm{s}$,

${ }^{a}$ http: //www.sdfast.com 

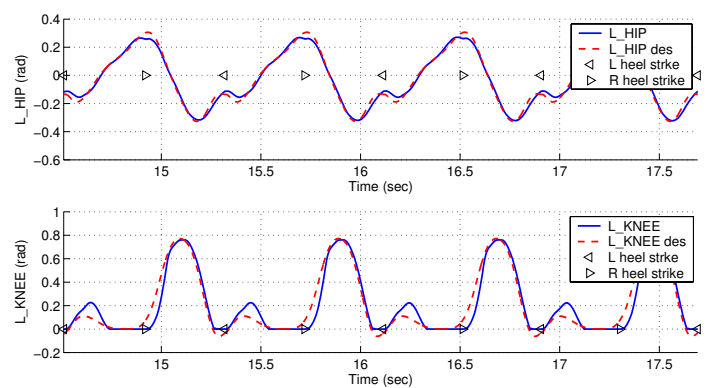

Fig. 3. Joint trajectories for the left leg and heel strike timing for four periods ( 8 steps) of walking (simulation).
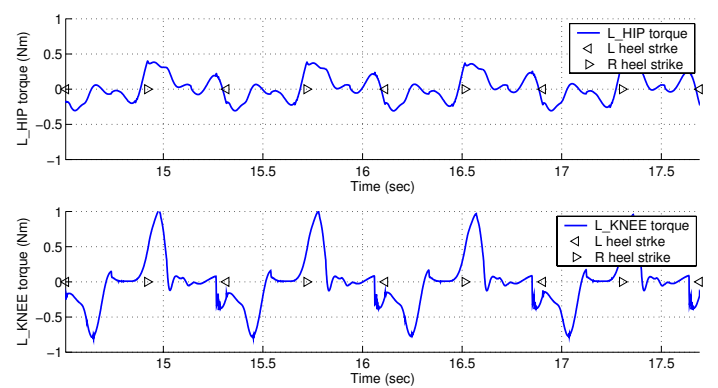

Fig. 4. Torque command to the left hip and knee joints for four periods ( 8 steps) of walking (simulation).

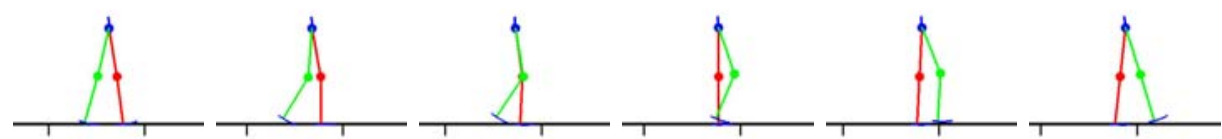

Fig. 5. Snapshots of walking simulation for one step at 15 frames/sec $(1$ frame $\approx 66 \mathrm{msec})$

which roughly corresponds to the natural frequency of the swing leg modelled as a simplified linear pendulum, using the proposed adaptation law.

\subsection{Robustness Against External Perturbations}

In this section, we numerically evaluate robustness of walking with the proposed algorithm against external perturbations by pushing the robot forward and backward with external forces during walking. Forces are applied for a duration of 0.1 

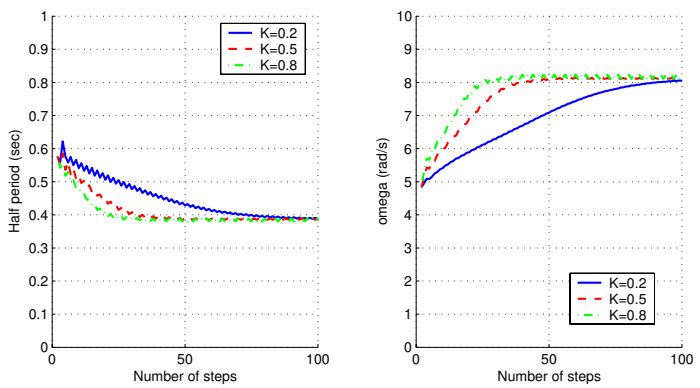

Fig. 6. Frequency adaptation of walking via entrainment (simulation). Left: adaptation of period. Right: learning curve of the frequency of the CPG.

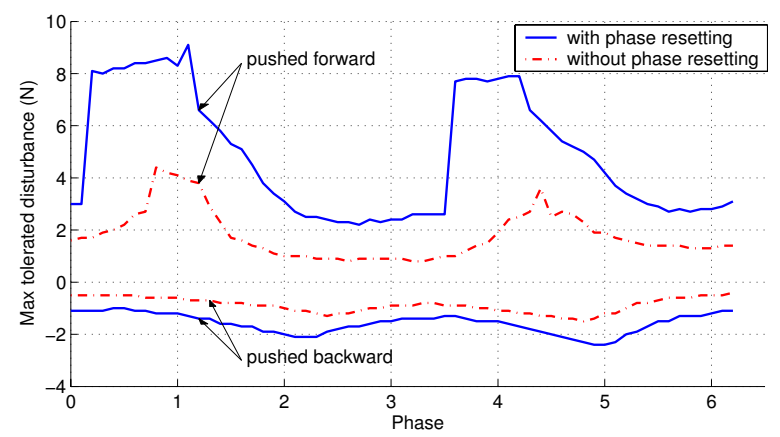

Fig. 7. Numerical evaluation of the robustness of walking against external perturbations. In the simulations, the robot is pushed forward and backward by perturbation forces for a duration of $0.1 \mathrm{sec}$ at difference timing during a single step. The plot shows the magnitude of the maximum external forces which the robot tolerated. This plot demonstrates that the robot could cope with much larger disturbances with phase resetting compared to the case without phase resetting.

sec at different timing during a single step (at an interval of $0.1 \mathrm{rad}$ from 0 to $2 \pi$ of the phase of the reference oscillator). We judge that the robot could tolerate the perturbation if the robot continues to walk over 30 steps after the disturbance is applied. Figure 7 shows the magnitude of the maximum external forces which the robot tolerated. When a forward perturbing force is applied, the robot could cope with up to $9.1 \mathrm{~N}(\max )$ at $\phi=1.1 \mathrm{rad}$, and $2.2 \mathrm{~N}(\min )$ at $\phi=2.7 \mathrm{rad}$ of the perturbing forces. When a backward perturbing force is applied, the robot could cope with up to $-2.4 \mathrm{~N}(\max )$ at $\phi=4.9 \mathrm{rad}$ and $-1.0 \mathrm{~N}(\min )$ at $\phi=0.4$ and $\phi=0.5 \mathrm{rad}$ of the perturbing forces. In contrast, without phase resetting, the robot only could cope with much smaller disturbances, as indicated by the plot in Fig. 7, for example, the robot only tolerated up to $3.9 \mathrm{~N}$ of the forward perturbing force applied at $\phi=1.1$. On average, with phase resetting, the robot tolerated up to 


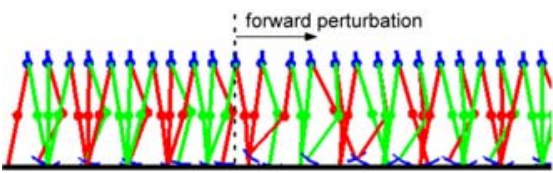

(a) with phase resetting

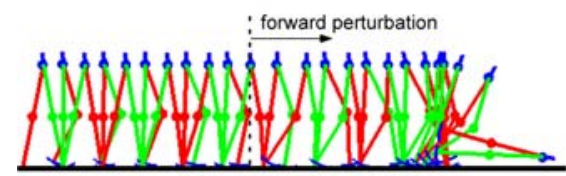

(b) without phase resetting

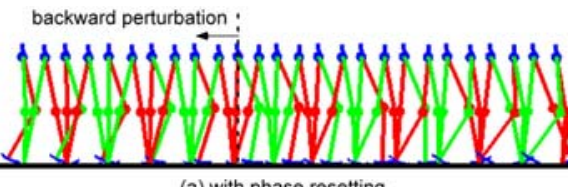

(a) with phase resetting

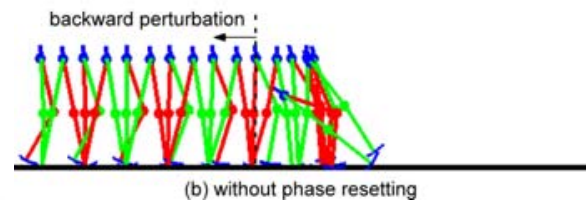

Fig. 8. Examples of the response against forward and backward perturbations. Left: the robot is pushed forward by a perturbation force of $6.0 \mathrm{~N}$ for a duration of $0.1 \mathrm{sec}$ at $\phi=0.5$. Right: the robot is pushed backward by a perturbation force of $-2.0 \mathrm{~N}$ for a duration of 0.1 sec at $\phi=2.0$. (a) with phase resetting, the robot is able to reject the disturbance and continue to walk. (b) without phase resetting, the robot falls over after the perturbation is applied.

$4.83 \mathrm{~N}$ and $-1.58 \mathrm{~N}$ of the forward and backward perturbing forces respectively. However, without phase resetting, the robot tolerated only up to $1.79 \mathrm{~N}$ and -0.88 $\mathrm{N}$ of the forward and backward perturbing forces respectively.

Figure 8 (left) depicts an example of the response against forward perturbations. The robot is pushed forward by a perturbation force of $6.0 \mathrm{~N}$ for a duration of $0.1 \mathrm{sec}$ at $\phi=0.5$. Figure 8 (right) depicts an example of the response against backward perturbations. The robot is pushed backward by a perturbation force of $-2.0 \mathrm{~N}$ for a duration of $0.1 \mathrm{sec}$ at $\phi=2.0$. The results in Fig. 8 (a) show that with phase resetting, the robot is able to reject the disturbance and continue to walk. In contrast, the results in Fig. 8 (b) show that without phase resetting, the robot falls over shortly after the perturbation is applied. The simulation results demonstrate the effectiveness of phase resetting to achieve robust walking against disturbance.

\section{Experimental Evaluations}

We implemented the proposed control framework on our biped robot described in Section 2. In the experiments our initial attempt to achieve biped locomotion using the human demonstrated trajectory was not successful. This was largely due to mechanical limitation of the experimental system and discrepancy in the ground contact condition between simulations and experiments. Thus, we used another target trajectory which was experimentally obtained from an actual trajectory of successful robot locomotion using a state machine controller. The state machine controller is designed to coordinate the leg movements with the physical state of the legged system based on the idea presented in ${ }^{28}$. To initiate locomotion in the experiments, we first suspended the robot with the legs swinging in the air, and then placed the robot on the ground manually. Thus, the initial condition of each run was not consistent, and occasionally the robot could not start walking or fell over 


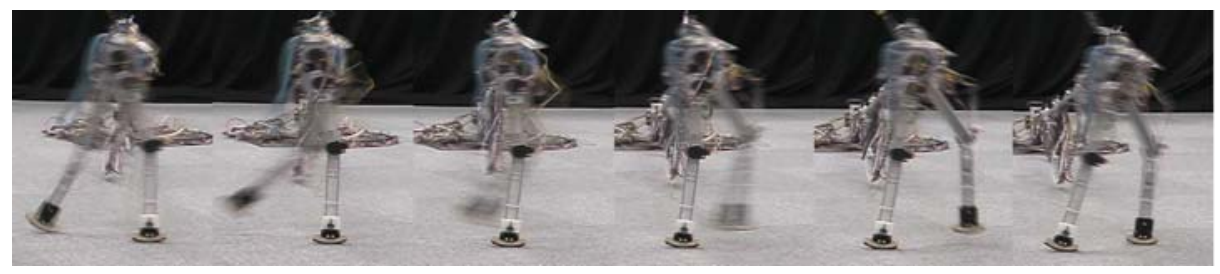

Fig. 9. Snapshots of a walking experiment on a flat surface (carpet) for a single step at 15 frames/sec $(1$ frame $\approx 66 \mathrm{msec})$
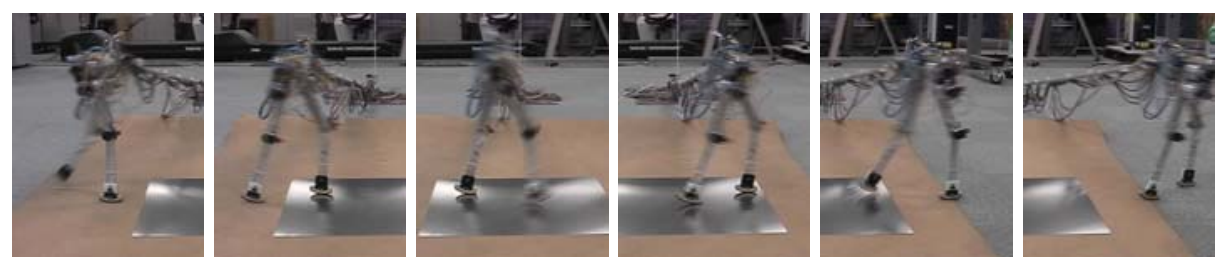

Fig. 10. Snapshots of a walking experiment over surfaces with different friction properties.

after a couple of steps when the timing was not appropriate. In this experiment, the initial frequency of the oscillator was set to $\omega=5.71 \mathrm{rad} / \mathrm{s}$ (period of oscillation is $1.1 \mathrm{sec}$ ), and the adaptation gain in (12) was decreased according to an annealing procedure $K=\frac{K_{0}}{n}$, where $K_{0}=0.05$ and $n$ is the number of steps, as it is needed in most gradient descent procedure. We introduced an offset $\alpha$ for phase resetting

$$
\dot{\phi}_{\text {ref }}=\hat{\omega}^{n}+\delta\left(t-t_{\text {heel strike }}\right)\left(\phi_{\text {heel strike }}^{\text {robot }}-\phi_{\text {ref }}+\alpha\right)
$$

to adjust the timing of foot contact, where $\alpha$ is chosen to be $\alpha=0.8 \mathrm{rad}$. These parameters are determined empirically. Note that phase resetting with an offset effectively changes the period of oscillation. Figure 9 presents snapshots of a walking experiment on a flat surface (carpet). Stepping period for a typical walking experiment was around $0.37 \mathrm{sec}$ as a result of frequency adaptation, and walking velocity was about $0.51 \mathrm{~m} / \mathrm{s}(1.87 \mathrm{~km} / \mathrm{h})$.

Robustness of the proposed algorithm is evaluated by testing walking over surfaces with different friction properties such as carpet, cork sheet ( $3 \mathrm{~mm}$ thick) and a metal plate ( $2 \mathrm{~mm}$ thick). Figure 10 shows an experimental result of walking over these different surfaces. In Fig. 11, the metal plate was placed so that the inclination of the slope slightly changes like a seesaw when the robot walks over it (the height of the center is $7 \mathrm{~mm}$ ). Figures $12-14$ show the desired and actual joint trajectories, the torque command, the timing of heel strike, and the stepping period of this walking experiment over a see-saw like metal plate respectively. The stepping period is disturbed when walking over the see-saw like plate (around 15th step, see Fig. 14), however, it is recovered after passing it. The robot could deal with these 

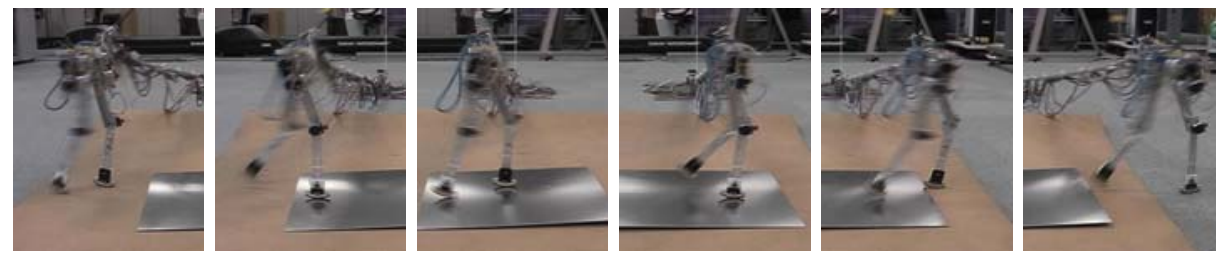

Fig. 11. Snapshots of a walking experiment over surfaces with different friction properties and a seesaw-like metal plate with a slight change in the slope. Also see the plots in Figs. 12-14 for joint trajectories, torque commands and stepping period for this experimental run, respectively.
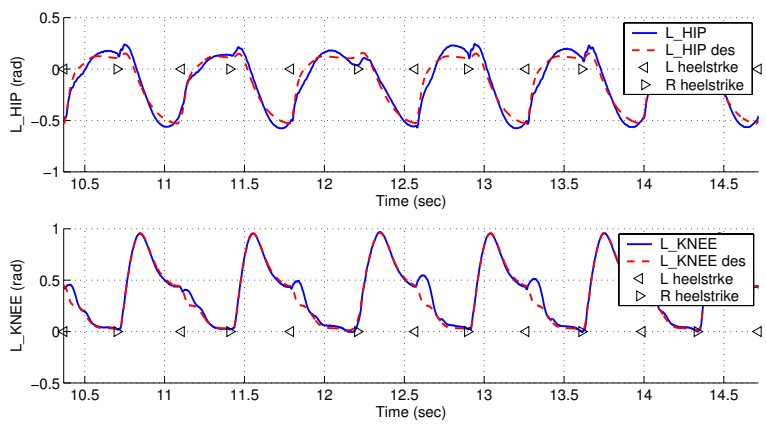

Fig. 12. Joint trajectories for the left leg and heel strike timing of the walking experiment over different surfaces with a see-saw like metal plate depicted in Fig. 11. These plots illustrate the joint trajectories of walking from 11th to 23rd steps in the corresponding plot in Fig. 14.
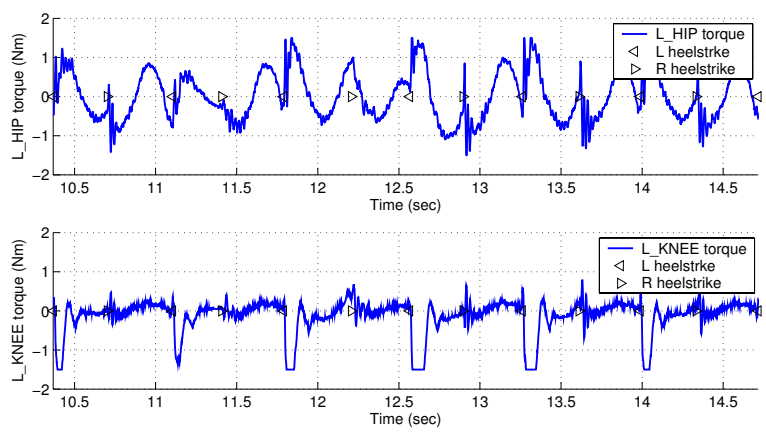

Fig. 13. Torque command to the left hip and knee joints of the walking experiment over different surfaces with a see-saw like metal plate depicted in Fig. 11.

environmental changes as the experimental results in Figs. 10 and 11 demonstrate.

Note that even if we use the learned trajectory from the actual robot walking 


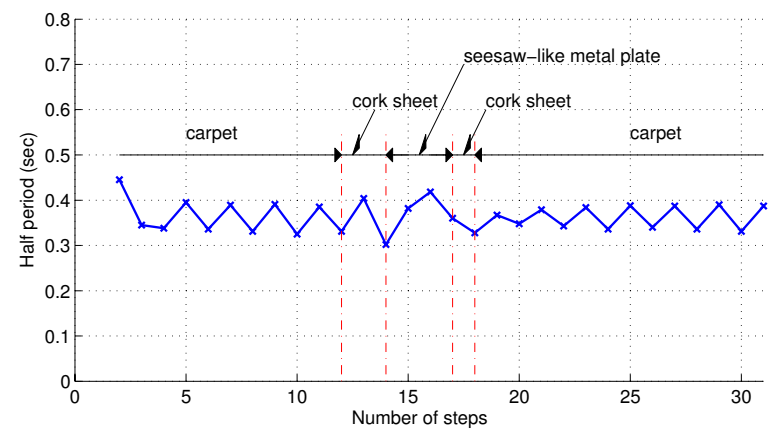

Fig. 14. Stepping period of a walking experiment over different surfaces with a see-saw like metal plate depicted in Fig. 11. The stepping period is disturbed when walking over the see-saw like metal plate, however, it is recovered after passing it.

pattern, the robot could not walk by just replaying it as a desired trajectory. Phase resetting using foot contact information was necessary. This implies that appropriate on-line adjustment of the phase of the CPG by sensory feedback from the environment is essential to achieve successful locomotion. In addition, empirically we found that the proposed controller achieved much more robust walking compared to the state machine based controller which we originally designed.

\section{Conclusion}

In this paper, we presented a method for learning biped locomotion from demonstration and its frequency adaptation using dynamical movement primitives. In the dynamical movement primitives, kinematic movement plans are described in a set of nonlinear differential equations with well-defined attractor dynamics, and demonstrated trajectories are learned using locally weighted regression. Specifically, we use rhythmic dynamical movement primitives based on coupled phase oscillators as a CPG, and introduced a frequency adaptation algorithm through interactions among the CPG, mechanical system and the environment motivated by the synchronization of coupled oscllators. Frequency adaptation of a CPG is beneficial when the desired frequency of the coupled system is not exactly known in advance. Numerical simulations and experimental result demonstrate the effectiveness of the proposed control algorithm to achieve steady state walking roughly at the natural frequency of the coupled system. Furthermore, we empirically evaluated robustness of walking with the proposed algorithm against external perturbations and environmental changes. The numerical and experimental results demonstrate the effectiveness of phase resetting contributes to achieve robustness of walking against disturbances.

Future work will address initiation and termination of walking, and on-line balance compensation. We will also consider collection of human's walking data under various behavioral conditions. In our current study, we used a simple phase reset- 
ting mechanism in which the phase of the CPG is forced to be reset to a specific value at the instance of heel strike regardless of the current phase of the CPG. In the future, we are interested in the generalization of the idea of phase resetting to determine phase dependent reaction against external perturbations such as recovery from stumbling by designing an appropriate phase resetting curve ${ }^{19}$. Formal mathematical analysis will be required to understand the principle of periodic stability of a limit cycle solution to the dynamics of a combined oscillator and mechanical system.

\section{Acknowledgements}

We would like to thank Auke Ijspeert at EPFL, Swiss Federal Institute of Technology, Lausanne and Seiichi Miyakoshi of the Digital Human Research Center, AIST, Japan, and Chris Atkeson at Carnegie Mellon University for valuable discussions and helpful comments on this work.

This research was supported in part by National Science Foundation grants ECS0325383, IIS-0312802, IIS-0082995, ECS-0326095, ANI-0224419, a NASA grant AC\#98-516, an AFOSR grant on Intelligent Control, the Communications Research Laboratory of Japan, the ERATO Kawato Dynamic Brain Project funded by the Japan Science and Technology Agency, and the ATR Computational Neuroscience Laboratories.

\section{References}

1. H. Miyamoto, S. Schaal, F. Gandolfo, Y. Koike, R. Osu, E. Nakano, Y. Wada, and M. Kawato. A kendama learning robot based on bi-directional theory. Neural Networks, 9:1281-1302, 1996.

2. Hiroyuki Miyamoto and Mitsuo Kawato. A tennis serve and upswing learning robot based on bi-directional theory. Neural Networks, 11:1317-1329, 1998.

3. Stefan Schaal. Is imitation learning the route to humanoid robots? Trends in Cognitive Sciences, 3(6):233-242, 1999.

4. Auke Ijspeert, Jun Nakanishi, and Stefan Schaal. Learning attractor landscapes for learning motor primitives. In S. Becker, S. Thrun, and K Obermayer, editors, Advances in Neural Information Processing Systems 15, pages 1547-1554. MIT-Press, 2003.

5. Kiyoshi Matsuoka. Sustained oscillatons generated by mutually inhibiting neurons with adaptation. Biologial Cybernetics, 52:367-376, 1985.

6. G. Taga, Y. Yamaguchi, and H. Shimizu. Self-organized control of bipedal locomotion by neural oscillators in unpredictable environment. Biological Cybernetics, 65:147-159, 1991.

7. Seiichi Miyakoshi, Gentaro Taga, Yasuo Kuniyoshi, and Akihiko Nagakubo. Three dimensional bipedal stepping motion using neural oscillators - towards humaniod motion in the real world. In Proceedings of the IEEE/RSJ International Conference on Intelligent Robots and Systems, pages 84-89, 1998.

8. Kazunori Hase and Nobutoshi Yamazaki. Computational evolution of human bipedal walking by a neuro-musculo-skeletal model. Artificial Life and Robotics, 3:133-138, 1999.

9. Gen Endo, Jun Morimoto, Jun Nakanishi, and Gordon Cheng. An empirical explo- 
ration of a neural oscillator for biped locomotion. In Proceedings of the IEEE International Conference on Robotics and Automation, 2004. (to appear).

10. Yasuhiro Fukuoka, Hiroshi Kimura, and Avis H. Cohen. Adaptive dynamic walking of a quadruped robot on irregular terrain based on biological concepts. International Journal of Robotics Research, 22(3-4):187-202, 2003.

11. Seiichi Miyakoshi, Masaki Yamakita, and Katsuhisa Furuta. Juggling control using neural oscillators. In Proceedings of the IEEE/RSJ International Conference on Intelligent Robots and Systems, pages 1186-1193, 1994.

12. S. Kotosaka and S Schaal. Synchronized robot drumming by neural oscillator. In Proceedings of the International Symposium on Adaptive Motion of Animals and Machines, 2000.

13. Matthew M. Williamson. Neural control of rhythmic arm movements. Neural Networks, 11:1379-1394, 1998.

14. E. Klavins and D. E. Koditschek. Phase regulation of decentralized cyclic robotic systems. International Journal of Robotics Research, 21:257-275, 2002.

15. Satoshi Ito, Hideo Yuasa, Zhi wei Luo, Masami Ito, and Dai Yanagihara. A mathematical model of adaptation in rhythmic motion to environmental changes. In Proceedings of the IEEE International Conference on Systems, Man and Cybernetics, pages 275280, 1997.

16. Katsuyoshi Tsujita, Kazuo Tsuchiya, and Ahmet Onat. Adaptive gait pattern control of a quadruped locomotion robot. In Proceedings of the IEEE/RSJ International Conference on Intelligent Robots and Systems, pages 2318-2325, 2001.

17. Kazuo Tsuchiya, Shinya Aoi, and Katsuyoshi Tsujita. Locomotion control of a biped locomotion robot using nonlinear oscillaotrs. In Proceedings of the IEEE/RSJ International Conference on Intelligent Robots and Systems, pages 1745-1750, 2003.

18. Jan Peters, Sethu Vijayakumar, and Stefan Schaal. Reinforcement learning for humanoid robotics. In Proceedings of the Humanoids2003, Third IEEE-RAS International Conference on Humanoid Robots, 2003.

19. Mitsuo Kawato. Transient and steady state phase response curves of limit cycle oscillators. Journal of Mathematical Biology, 12:13-30, 1981.

20. Taiga Yamasaki, Taishin Nomura, and Shunsuke Sato. Possible functional roles of phase resetting during walking. Biological Cybernetics, 88(6):468-496, 2003.

21. Jun Morimoto, Garth Zeglin, and Christopher G. Atkeson. Minimax differential dynamic programming: Application to a biped walking robot. In Proceedings of the IEEE/RSJ International Conference on Intelligent Robots and Systems, pages 19271932, 2003.

22. S. Schaal and C. G. Atkeson. Constructive incremental learning from only local information. Neural Computation, 10(8):2047-2084, 1998.

23. Auke Jan Ijspeert. A connectionist central pattern generator for the aquatic and terrestrial gaits of a simulated salamander. Biological Cybernetics, pages 331-348, 2001.

24. Yoshiki Kuramoto. Chemical Oscillations, Waves, and Turbulence. Springer-Verlag, 1984.

25. Kosuke Sekiyama, Jun Nakanishi, Isao Takagawa, Toshimitsu Higashi, and Toshio Fukuda. Self-organizing control of urban traffic signal network. In Proceedings of the IEEE International Conference on Systems, Man and Cybernetics, pages 2481-2486, 2001.

26. Jun Nakanishi, Jun Morimoto, Gen Endo, Gordon Cheng, Stefan Schaal, and Mitsuo Kawato. Learning from demonstration and adaptation of biped locomotion with dynamical movement primitives. Workshop on Robot Programming by Demonstra- 
tion, IEEE/RSJ International Conference on Intelligent Robots and Systems, 2003. manuscript available at www.cns.atr.jp/ jun/int_pub.html.

27. Yoshihiro Ehara and Sumiko Yamamoto. Introduction to Body-Dynamics-Analysis of Gait and Gait Initiation. Ishiyaku Publishers, 2002. in Japanese.

28. Jessica K. Hodgins. Biped gait transitions. In Proceedings of the IEEE International Conference on Robotics and Automation, pages 2092-2097, 1991. 